\title{
Surgical resection of vasoactive intestinal peptideoma with hepatic metastasis aids symptom palliation: A case report
}

\author{
XIAOMEI ZHANG ${ }^{1}$, LINGLI ZHOU ${ }^{2}$, YING LIU ${ }^{3}$, WEI LI $^{4}$, HONGKAI GAO $^{5}$, \\ YUNAN WANG ${ }^{3}$, BAOTING YAO $^{3}$, DAMING JIANG $^{6}$ and PEIJUN HU ${ }^{7}$ \\ ${ }^{1}$ Department of Endocrinology, Peking University International Hospital, Beijing 102206; \\ ${ }^{2}$ Department of Endocrinology, Peking University People's Hospital, Beijing 100044; ${ }^{3}$ Department of Internal Medicine, \\ First Hospital, Dandong, Liaoning 118000; ${ }^{4}$ Department of Surgical Medicine, First Hospital, Dandong, Liaoning 118000; \\ ${ }^{5}$ Department of Surgical Medicine, General Hospital of the Chinese People's Armed Police Forces, Beijing 100039; \\ ${ }^{6}$ Department of Internal Medicine, Central Hospital, Dandong, Liaoning 118000; ${ }^{7}$ Department of Gastroenterology, \\ Beijing First Hospital of Integrated Chinese and Western Medicine, Beijing 100026, P.R. China
}

Received June 10, 2014; Accepted February 5, 2015

DOI: $10.3892 /$ etm.2016.3019

\begin{abstract}
Vasoactive intestinal peptideoma (VIPoma) is a rare pancreatic endocrine tumor associated with a well-defined clinical syndrome characterized by watery diarrhea, hypokalemia and metabolic acidosis. In adults, VIPoma is most commonly found in the pancreas, with $80 \%$ of the tumors occurring in the body and tail and $20 \%$ occurring in the pancreatic head. VIPomas can represent a significant diagnostic challenge due to their nonspecific clinical presentation, which can result in the misdiagnosis of a VIPoma as another condition, such as laxative overdose or a carcinoid secreting tumor. Surgical clearance of the tumor is the first-line treatment, even in cases with metastasis. The present study describes the case of a patient who presented with chronic watery diarrhea and hypokalemia due to a tumor in the pancreatic head, which was confirmed to contain immunoreactive vasoactive intestinal polypeptide via immunohistochemistry. A hepatic metastasis lesion was diagnosed following computed tomography. Stable control of symptoms was achieved after surgery and drug treatment. The study additionally reviews the clinical, histological, radiological and diagnostic features of the condition, as well as the therapeutic modalities that can be used to treat VIPoma in the pancreatic head with hepatic metastasis.
\end{abstract}

Correspondence to: Dr Peijun Hu, Department of Gastroenterology, Beijing First Hospital of Integrated Chinese and Western Medicine, Jin Tai Road, 13th Chaoyang, Beijing 100026, P.R. China

E-mail: xiaoxiaopei@sina.com

Abbreviations: WDHA, watery diarrhea associated with hypokalemia and achlorhydria; VIP, vasoactive intestinal polypeptide; CT, computed tomography; MRI, magnetic resonance imaging; PET, positron emission tomography

Key words: vasoactive intestinal peptideoma, metastasis

\section{Introduction}

The clinical symptoms that accompany vasoactive intestinal peptideoma (VIPoma) most commonly include watery diarrhea, hypokalemia and achlorhydria (or metabolic acidosis); this collection of symptoms is also known as WDHA syndrome. WDHA syndrome was first described by Verner and Morrison in 1958 (1), and has been assumed to be caused by the hypersecretion of vasoactive intestinal polypeptide (VIP) (2). In adults, this tumor is most commonly found in the pancreas, with $80 \%$ of the tumors occurring in the body and tail of pancreas and $20 \%$ occurring in the pancreatic head (3). These tumors are usually solitary and $>3 \mathrm{~cm}$ in diameter. Between 50-60\% of pancreatic VIPomas have already developed metastases at the point of diagnosis, primarily in the liver and lymph nodes (4). The usual methods of treating VIPoma are surgical excision, peptide receptor radionuclide therapy, streptozotocin-based chemotherapy, ablation, hepatic artery embolization, liver transplantation and adjuvant therapy, depending on the condition of patient. The median overall survival of pancreatic endocrine tumors is 38 months, with localized, regional and distant islet cell carcinoma survival durations of 124, 70 and 23 months, respectively (5). The present study describes the case of a patient who presented with chronic watery diarrhea and hypokalemia due to a tumor in the pancreatic head, which was immunohistochemically confirmed to contain immunoreactive VIP and diagnosed as a metastatic hepatic lesion through computed tomography (CT). Written informed consent for publication was obtained from the patient.

\section{Case report}

A 65-year-old male, presenting with a six-month history of profuse watery diarrhea, anorexia, vomiting, a $5-\mathrm{kg}$ weight loss and extreme weakness, was admitted to the First Hospital of Dandong (Liaoning, China), in 2011. The patient had $\geq 10$ watery bowel movements per day without blood 


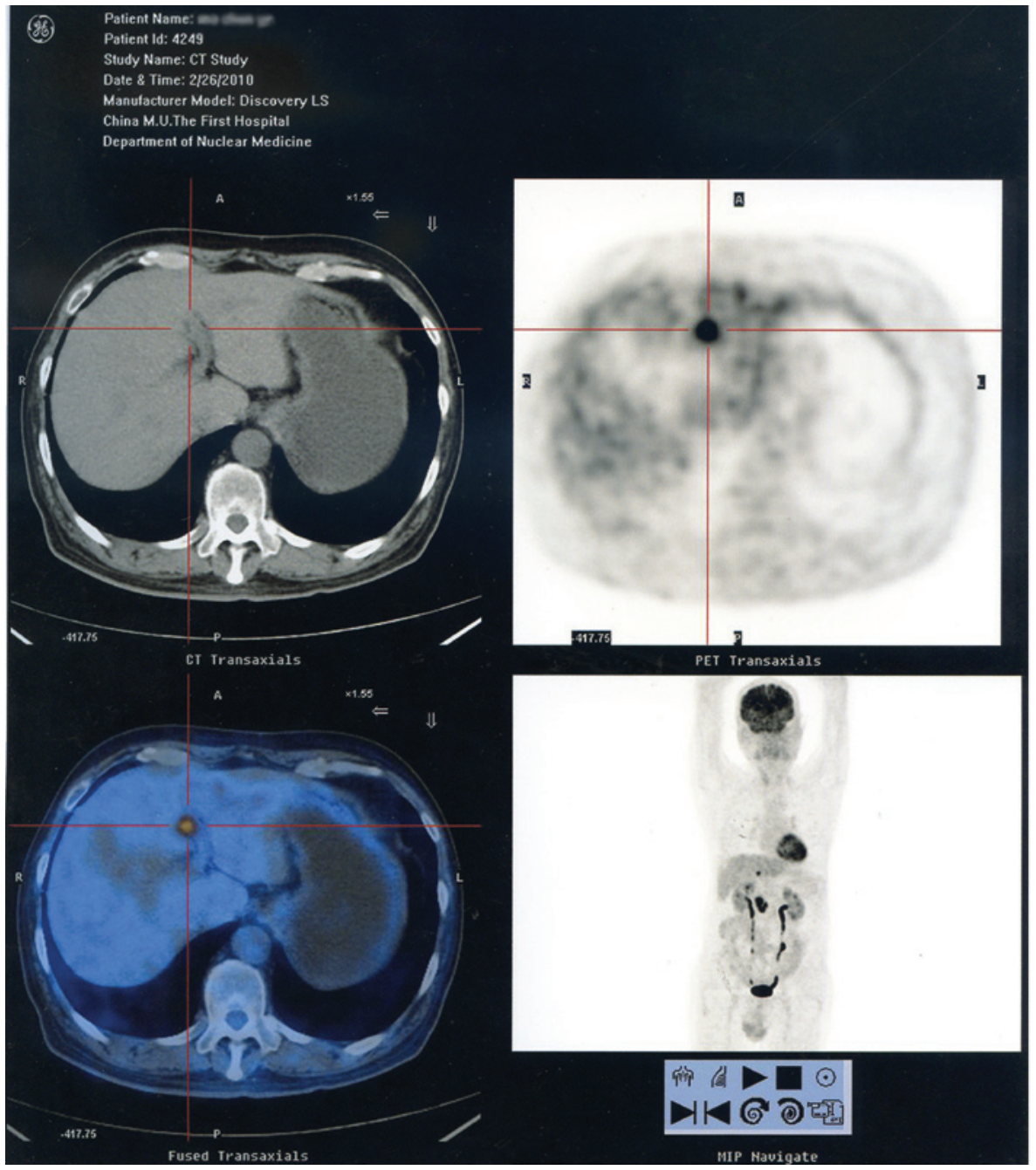

Figure 1. PET-CT showed ${ }^{18} \mathrm{~F}$-fluorodeoxyglucose uptake in the shadow of the pancreas, with the corresponding regions of the CT showing a soft tissue density shadow. CT, computed tomography; PET, positron emission tomography.

or mucus. The symptoms were not relieved following oral levofloxacin administration. Physical examination revealed a dehydrated appearance and generalized weakness. The patient's blood pressure was $85 / 55 \mathrm{mmHg}$ and his heart rate was 92 beats $/ \mathrm{min}$. Assessment of the breath sounds revealed rough lung breath sounds with occasional wheezing; however, cardiac auscultation was normal. No tenderness was present in the liver, kidney or other areas of the abdomen. Active bowel sounds were found with abdominal auscultation. The patient had a previous history of bronchial asthma for 20 years and was diagnosed with hyperthyroidism 10 years previously. This hyperthyroidism was subsequently cured. The rest of the patient's history, and that of his family, was not noteworthy.

Laboratory examination revealed that all the biochemical tests, including the hemoglobin level, white blood cell count, urinalysis, and renal and liver function, were normal. Microbiological and parasitological examinations of the feces yielded no positive findings. The blood cell dissemination was $39 \mathrm{mmol} / \mathrm{l}$. Plasma sodium, chloride, phosphate, calcium, urine amylase and fasting blood glucose levels were normal; however, marked hypokalemia $(2.26 \mathrm{mmol} / \mathrm{l})$ was noted. The level of the tumor marker carcinoembryonic antigen (CEA) was
$3.63 \mathrm{ng} / \mathrm{ml}$. The majority of the indices of thyroid function were within the normal ranges, with the exception of the levels of free thyroxine $(18.15 \mathrm{pmol} / \mathrm{l})$, which were a little higher. A bilateral adrenal CT scan, colonoscopy, double-contrast enteroclysis and an abdominal ultrasound were performed, but no meaningful findings were obtained. Gastroscopy showed chronic superficial gastritis. The electrocardiograph showed prominent $U$ waves consistent with hypokalemia. An abdominal CT scan showed a mass with a 6-cm diameter in the pancreatic head.

Based on these results, the patient was diagnosed with hypokalemia and diarrhea. Fluid infusions were initiated and the patient was administered $6 \mathrm{~g}$ potassium per day and oral anti-inflammatory drugs; however, the hypokalemia and diarrhea reoccurred following the cessation of the drug administration. The chronic diarrhea and persistent hypokalemia, and the presence of a mass in the pancreas, suggested that a vasodilatory intestinal peptide-secreting tumor could not be excluded.

Experimental treatment of $0.1 \mathrm{mg}$ octreotide twice per day by intravenous infusion was subsequently carried out. The patient showed significant relief from the diarrhea and nausea following the first day of octreotide treatment. One week later, the nausea and other discomfort were also reduced significantly, and the octreotide was then terminated. The 


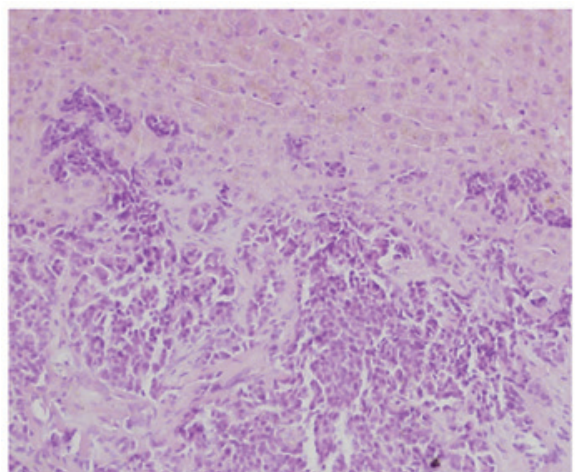

HE

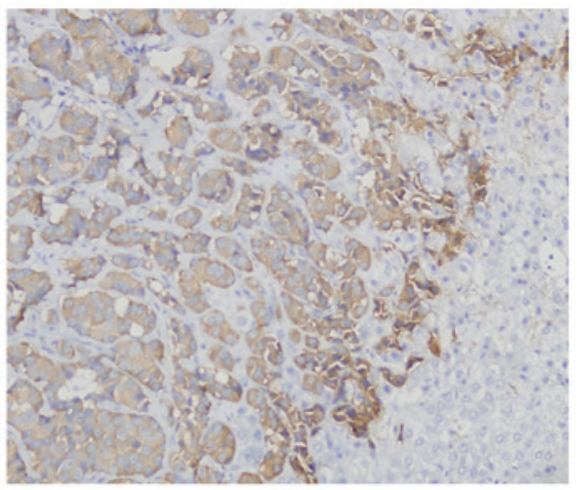

SYN

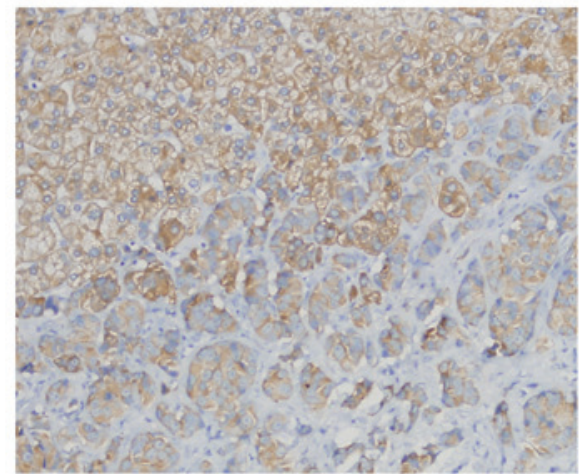

CK-18

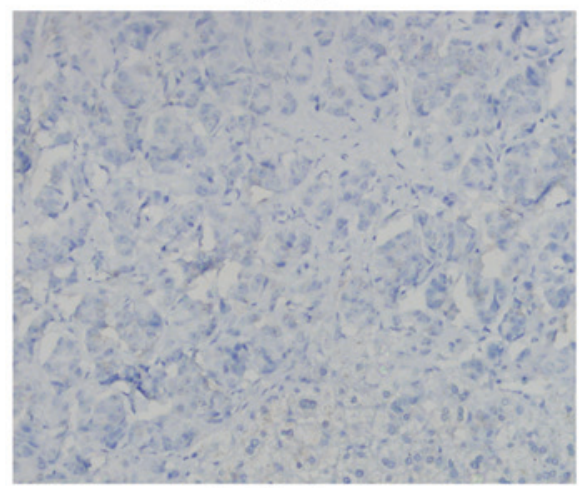

Calcitonin

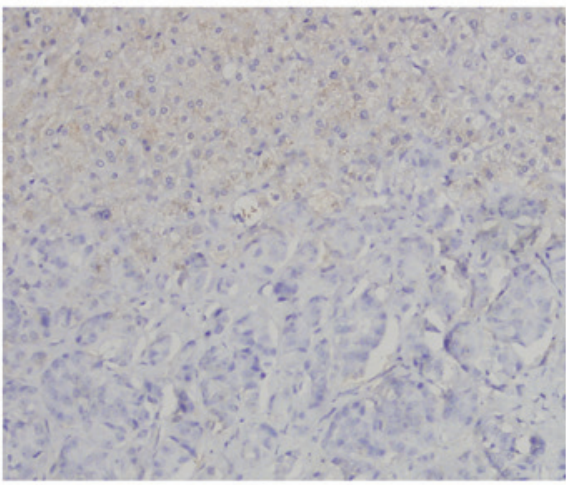

AFP

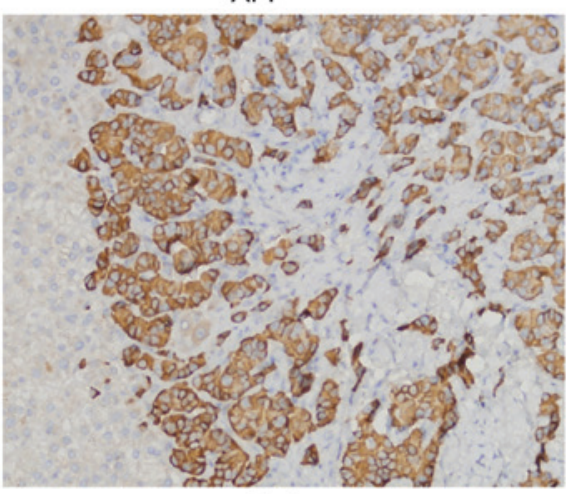

$\mathrm{CgA}$

Figure 2. Immunohistochemistry results showed AFP (-), calcitonin (-), CgA (+), CK18 (+) and Syn (+). HE, hematoxylin and eosin; AFP, $\alpha$-fetoprotein; CgA, chromogranin A; CK18, cytokeratin 18; Syn, synaptophysin. Magnification, x10.

condition reoccurred following the suspension of the octreotide; however, re-administration of the octreotide still produced positive results. The effectiveness of the octreotide on the diarrhea made the diagnosis of a VIPoma highly likely. A blood sample was therefore sent to the Chinese Medical University Affiliated Hospital (Shenyang, China) to measure the VIP concentration. The diagnosis was confirmed by the high level of VIP (>600 pg/ml). ${ }^{18}$ F-fluorodeoxyglucose-positron emission tomography (PET) showed abnormal uptake at the same location as the pancreatic tumor revealed by CT. The maximum standardized uptake value of the lesion was 13.0 Hounsfield units (Fig. 1). The combination of the symptoms, response to octreotide and findings of the imaging studies prompted the decision to perform an exploratory laparotomy.

Multiple nodules in the liver were found during the surgery, which were considered to be hepatic metastasis. The pancreatic tumor, which measured $5.0 \times 4.0 \times 4.0 \mathrm{~cm}$, exhibited infiltration into the transverse mesocolon. The mass and the superior mesenteric vein invasion could not be separated. The intraoperative pathological biopsy diagnosis was VIPoma with liver metastasis. The tumor was excised from the pancreas.

The VIP level returned to the normal range following surgery. Oral potassium supplementation, octreotide and oral prednisolone were still required for symptom relief. During the one-and-a-half years after the surgery, the frequency of the diarrhea episodes was reduced to approximately four times per day.

The aforementioned symptoms became apparent once more one-and-a-half years after the surgery. Abdominal CT indicated that the pancreas exhibited a lack of uniform density, while the liver was normal in size and shape. The left and right hepatic

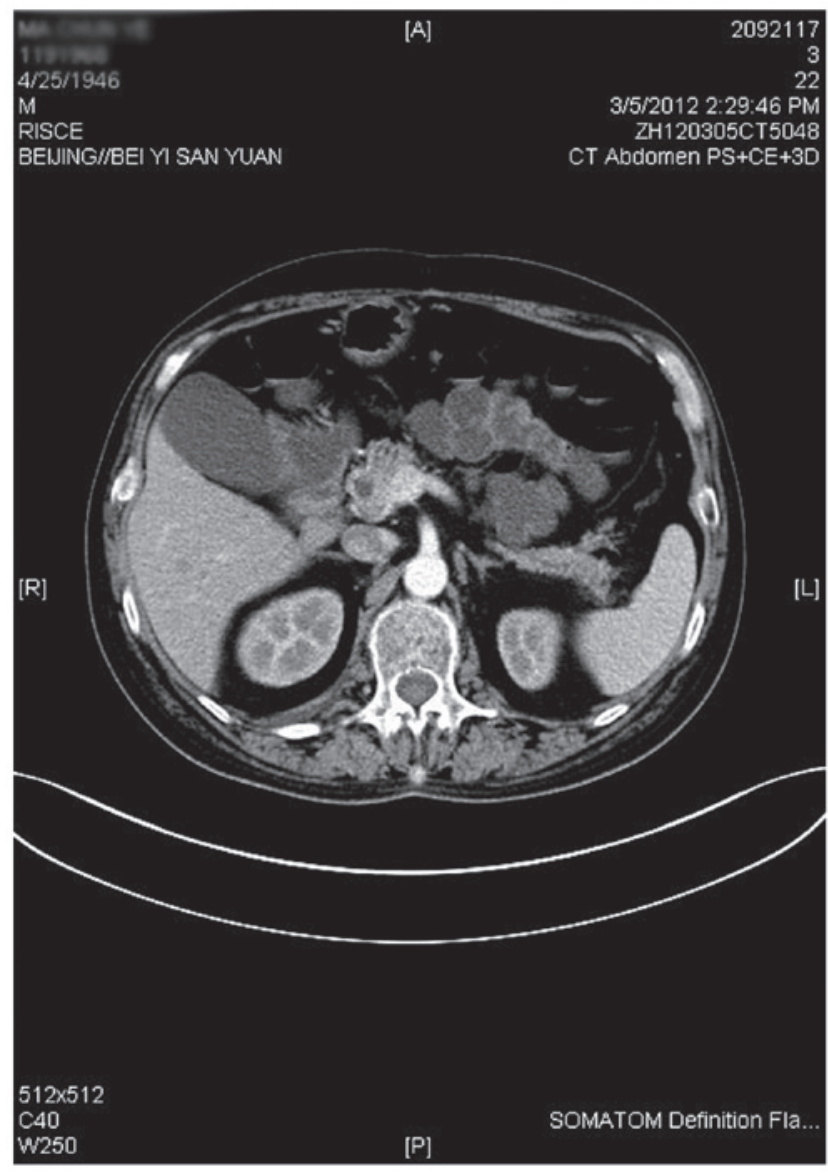

Figure 3. A large, circular, low-density liquid and gas shadow was found in the left lateral lobe of the liver, which indicated liver abscess. 
lobes showed multiple sizes of low-density nodules with CT values between 17 and $55 \mathrm{HU}$, which were considered to be liver metastasis. The abdominal lymph nodes were normal. Magnetic resonance imaging (MRI) confirmed the observations of the CT. Under general anesthesia, pancreatoduodenectomy, superior mesenteric vein partial resection and reconstruction, partial resection of the liver metastatic nodules (four nodules with a diameter $>0.5 \mathrm{~cm}$ in the left outer lobe, two in the left inner lobe, three in the right anterior lobe and one in the right posterior lobe) and liver surface metastasis nodule cauterization were performed successively. The pathological diagnosis was pancreatic mixed ductal-endocrine cancer. The lymph node biopsy showed cancer metastasis. The results of the immunohistochemistry were as follows (Fig. 2): $\alpha$-fetoprotein (AFP) (-), calcitonin (-), chromogranin A (CgA) (+), cytokeratin (CK) $18(+)$ and synaptophysin $(+)$. The patient was transferred to the intensive care unit following the surgery. Rehydration, anti-inflammatory agents and gastric acid and trypsin inhibitors were administered, and nutritional support was carried out. Persistent low levels of potassium and intermittent atrial fibrillation were noted following the surgery. The left hepatic abscess was found through abdominal CT examination (Fig 3). Subsequent to cardioversion, potassium supplementation and anti-infection therapy, the condition of the patient gradually stabilized. A follow-up at 18 months showed that the patient is still alive and has achieved partial control of his symptoms.

\section{Discussion}

Pancreatic VIP-secreting tumors are rare islet cell tumors associated with secretory diarrhea. The incidence of this type of neoplasm is estimated to be one per 10,000,000 individuals in the general population annually (2). A total of $90 \%$ of the VIPomas in adults originate from the pancreas (3). VIP is a 28-amino acid polypeptide with high homology in structure to secretin. Following the binding of VIP to receptors on the intestinal epithelial cells, adenylate cyclase and cyclic adenosine monophosphate production is activated. This leads to the secretion of water and electrolytes into the intestinal lumen (1). Patients typically present with chronic diarrhea and are diagnosed late due to the slow-growing nature of the tumor. It is difficult to find VIPomas when they are small. Symptomatic pancreatic VIPomas are usually $>3 \mathrm{~cm}$ in diameter. At the time of presentation, $>70 \%$ of patients have developed metastases (3).

The diagnosis of VIP-secreting tumors includes clinical symptoms and laboratory assays. The major clinical symptoms of VIP-secreting tumors include severe watery diarrhea, hypokalemia and acid-free or low levels of gastric acid secretion, which is referred to as WDHA syndrome or pancreatic cholera (1). A high volume of diarrhea is universal, and hypokalemia occurs in $70-100 \%$ of patients (2). Other symptoms include severe dehydration, gallbladder enlargement, intestinal pseudo-infarction, high blood sugar and high blood calcium levels. The patient in the present case report had watery diarrhea $>10$ times per day and exhibited persistent low blood potassium and gastric acid secretion levels, hypovolemic shock and gallbladder enlargement, all of which were diagnostically consistent with the disease. Laboratory assays in the diagnosis of the disease mainly include serum VIP, pancreatic polypeptide and $\mathrm{CgA}$ (2). In the present case, only the concentration of VIP was measured due to the limitations of the laboratory conditions. The plasma VIP concentration was found to measure $>600 \mathrm{ng} / \mathrm{l}$, while the accepted standard for plasma the VIP concentration in patients with VIP-secreting tumors is $\geq 200 \mathrm{ng} / \mathrm{l}$ (2). In addition to laboratory testing, CT and MRI have a sensitivity of $80-85 \%$, and functional PET imaging has a sensitivity of $\sim 97 \%$ (6). These imaging techniques are also used to determine whether metastases are present. VIP-secreting tumors are typically located in the pancreatic body and tail, and more than half of the clinical cases have a complete capsule (3). In the current case, the patient showed lesions in the pancreatic head, as detected by CT.

Considerable advances have been made in the management of VIPoma. The first line of treatment is surgical excision for patients with benign and non-metastatic disease; however, there is no accepted standard management for patients with metastatic disease. Surgery for tumor clearance, peptide receptor radionuclide therapy (6), streptozotocin-based chemotherapy (7), ablation (8), hepatic artery embolization (9), liver transplantation, sunitinib/everolimus (10) and adjuvant therapy, such as octreotide, interferon- $\alpha$ and glucocorticoids, are suggested, depending on the condition of patients. In the present case, the patient underwent surgeries to remove tumors on the pancreas and liver, respectively. The diarrhea improved following surgery and adjuvant treatment, and its frequency was reduced to four times a day as compared with 10 times preoperatively. The somatosatin analogue, octreotide, was administrated for symptomatic relief. Long-term application of the drug, however, may lead to drug resistance and the necessity for an increase in dosage $(11,12)$. Prednisone and indomethacin are also considered to be effective in alleviating the clinical symptoms; therefore, octreotide, at a dosage of $100 \mathrm{mg}$ daily, combined with $20 \mathrm{mg}$ prednisone daily were administered to the patient in this study to control the symptom of diarrhea and arrest the liver metastasis.

In conclusion, VIPoma is rare, and the disease has typically already metastasized at presentation. Considering VIPoma in patients with chronic diarrhea and hypokalemia would aid the early diagnosis of this disease. In total, $40 \%$ of patients with non-metastatic disease are likely to be cured by a complete resection of the tumor (13). Palliative surgery is indicated in advanced disease, followed by somatostatin analogue therapy. Somatostatin analogues improve hormone-mediated symptoms and reduce tumor volume; however, long-term application of octreotide is likely to lead to drug resistance and inhibit the secretion of insulin, growth hormone and glucagon, which is problematic. The use of corticosteroids has achieved successful responses in several cases $(14,15)$. The combined application of corticosteroids with octreotide may reduce the required dosage of octreotide, avoid the side-effects and ease the financial burden of the patient.

\section{References}

1. Verner JV and Morrison AB: Islet cell tumor and a syndrome of refractory watery diarrhea and hypokalemia. Am J Med 25: 374-380, 1958

2. Ghaferi AA, Chojnacki KA, Long WD, Cameron JL and Yeo CJ: Pancreatic VIPomas: subject review and one institutional experience. J Gastrointest Surg 12: 382-393, 2008. 
3. Perry RR and Vinik AI: Clinical review 72: diagnosis and management of functioning islet cell tumors. J Clin Endocrinol Metab 80: 2273-2278, 1995.

4. Soga J and Yakuwa Y: VIPoma/diarrheogenic syndrome: A statistical evaluation of 241 reported cases. J Exp Clin Cancer Res 17: 389-400, 1998.

5. Yao JC, Eisner MP, Leary C, Dagohoy C, Phan A, Rashid A, Hassan M and Evans DB: Population-based study of islet cell carcinoma. Ann Surg Oncol 14: 3492-3500, 2007.

6. Baum RP and Kulkarni HR: THERANOSTICS: from molecular imaging using Ga-68 labeled tracers and PET/CT to personalized radionuclide therapy - the Bad Berka experience. Theranostics 2: 437-447, 2012.

7. Moertel CG, Lefkopoulo M, Lipsitz S, Hahn RG and Klaassen D: Streptozocin-doxorubicin, streptozocin-fluorouracil or chlorozotocin in the treatment of advanced islet-cell carcinoma. N Engl J Med 326: 519-523, 1992.

8. Moug SJ, Leen E, Horgan PG and Imrie CW: Radiofrequency ablation has a valuable therapeutic role in metastatic VIPoma Pancreatology 6: 155-159, 2006.

9. Shaib W, Mitchell K and Saif MW: Amelioration of symptoms and reduction of VIP levels after hepatic artery chemoembolization in a patient with sandostatin resistant VIPoma. Yale J Bio Med 83: 27-33, 2010.
10. Strosberg JR, Cheema A and Kvols LK: A review of systemic and liver-directed therapies for metastatic neuroendocrine tumors of the gastroenteropancreatic tract. Cancer Control 18: 127-137, 2011.

11. Adam N, Lim SS, Ananda V and Chan SP: VIPoma syndrome: challenges in management. Singapore Med J 51: e129-e132, 2010.

12. Yasunami Y, Funakoshi A, Ryu S, et al: In vitro release of vasoactive intestinal polypeptide and pancreatic polypeptide from human VIPoma cells and its inhibition by somatostatin analogue (SMS 201-995). Surgery 115: 713-717, 1994.

13. Arnold R, Frank M and Kajdan U: Management of gastroenteropancreatic endocrine tumors: the place of somatostatin analogues. Digestion 55 (Suppl 3): 107-113, 1994.

14. Nguyen HN, Backes B, Lammert F, et al: Long-term survival after diagnosis of hepatic metastatic VIPoma: report of two cases with disparate courses and review of therapeutic options. Dig Dis Sci 44: 1148-1155, 1999.

15. Zimmermann K, Rapp T, Binder J, Frölich J and Bode JC: Vipoma. 9-year observations using currently available therapy methods. Dtsch Med Wochenschr 111: 298-301, 1986 (In German). 